\title{
ARTICLE \\ Xanthatin induces glioma cell apoptosis and inhibits tumor growth via activating endoplasmic reticulum stress-dependent CHOP pathway
}

\author{
Yu-yang $\mathrm{Ma}^{1,2}$, Ze-min $\mathrm{Di}^{1,2}$, Qing $\mathrm{CaO}^{1,2}$, Wen-shuang $\mathrm{Xu}^{1,2}$, Si-xing $\mathrm{Bi}^{1,2,3}$, Ji-shuang $\mathrm{Yu}^{1,2}$, Yu-jun Shen ${ }^{1,2}$, Yong-qiang $\mathrm{Yu}^{3}$, \\ Yu-xian Shen ${ }^{1,2}$ and Li-jie Feng ${ }^{1,2}$
}

Xanthatin is a natural sesquiterpene lactone purified from Xanthium strumarium L., which has shown prominent antitumor activity against a variety of cancer cells. In the current study, we investigated the effect of xanthatin on the growth of glioma cells in vitro and in vivo, and elucidated the underlying mechanisms. In both rat glioma C6 and human glioma U251 cell lines, xanthatin (1-15 $\mu \mathrm{M})$ dose-dependently inhibited cell viability without apparent effect on the cell cycle. Furthermore, xanthatin treatment dosedependently induced glioma cell apoptosis. In nude mice bearing C6 glioma tumor xenografts, administration of xanthatin (10, 20, $40 \mathrm{mg} \cdot \mathrm{kg}^{-1} \cdot \mathrm{d}^{-1}$, ip, for 2 weeks) dose-dependently inhibited the tumor growth, but did not affect the body weight. More importantly, xanthatin treatment markedly increased the expression levels of the endoplasmic reticulum (ER) stress-related markers in both the glioma cell lines as well as in C6 xenografts, including glucose-regulated protein 78, C/EBP-homologous protein (CHOP), activating factor 4 , activating transcription factor 6 , spliced X-box binding protein-1, phosphorylated protein kinase R-like endoplasmic reticulum kinase, and phosphorylated eukaryotic initiation factor 2a. Pretreatment of C6 glioma cells with the ER stress inhibitor 4-phenylbutyric acid (4-PBA, $7 \mathrm{mM}$ ) or knockdown of CHOP using small interfering RNA significantly attenuated xanthatininduced cell apoptosis and increase of proapoptotic caspase-3. These results demonstrate that xanthatin induces glioma cell apoptosis and inhibits tumor growth via activating the ER stress-related unfolded protein response pathway involving CHOP induction. Xanthatin may serve as a promising agent in the treatment of human glioma.

Keywords: glioma; xanthatin; apoptosis; endoplasmic reticulum stress; CHOP; 4-phenylbutyric acid; rat glioma C6 cell line; human glioma U251 cell line

Acta Pharmacologica Sinica (2020) 41:404-414; https://doi.org/10.1038/s41401-019-0318-5

\section{INTRODUCTION}

Glioma is a primary neuroepithelial tumor of the central nervous system characterized by an extremely aggressive clinical phenotype $[1,2]$. Despite recent advances in standard therapy, including maximal surgical excision followed by radiotherapy with concomitant and adjuvant chemotherapy, the treatment of glioma remains extremely unfavorable [1,3]. Therefore, the development of novel strategies and the discovery of new drugs for glioma therapy are essential.

To date, natural products have been the primary source of chemotherapeutics and provided abundant candidates with promising antitumor activities. Xanthatin is a natural bioactive sesquiterpene lactone isolated from the aerial part of Xanthium strumarium $L$. (Asteraceae), a native herb commonly used in China $[4,5]$. Previous studies demonstrated that xanthatin exhibits a wide variety of bioactivities, including anticancer $[6,7]$, antiangiogenesis [8-10], antiviral [10], antiinflammatory [11], antifungal [12, 13], antibacterial [14], antitrypanosome [11], and antileishmanial [13] activities. In addition, xanthatin was reported to suppress proliferation and induce apoptosis in a wide variety of cancer cell culture systems, including non-small cell lung cancer cells (A549 and H522 cells), MKN-45 human gastric carcinoma cells, MDA-MB-231 human breast cancer cells, B16-F10 murine melanoma cells, and L1210 mouse leukemia cells [9, 10, 15-23]. Recently, we dynamically monitored the antitumor growth effect of xanthatin in glioma-bearing mice using conventional magnetic resonance imaging (MRI) and dynamic contrast-enhanced MRI [24]. However, no detailed studies have reported the effect of xanthatin on glioma cell apoptosis, and the underlying mechanisms of its antitumor activity have yet to be elucidated.

The endoplasmic reticulum (ER) is a fundamental cellular organelle responsible for a variety of physiological processes, including the synthesis, folding, and posttranslational modification of the majority of membrane and secretory proteins and calcium storage $[25,26]$. The accumulation of unfolded or misfolded proteins in the ER results in ER stress that triggers cytoprotective signaling pathways, termed the unfolded protein response (UPR), to restore and maintain homeostasis in the ER [26]. If the UPR is

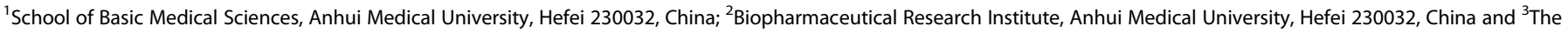
First Affiliated Hospital of Anhui Medical University, Hefei 230032, China

Correspondence: Yu-xian Shen (shenyx@ahmu.edu.cn) or Li-jie Feng (fenglijie1128@sina.com)

These authors contributed equally: Yu-yang Ma, Ze-min Di
}

Received: 23 April 2019 Accepted: 9 October 2019

Published online: 7 November 2019 
unable to correct the balance of ER stress, the cellular apoptotic machinery may be triggered, ultimately leading to cell death $[25,27,28]$. A number of recent studies have revealed that certain chemotherapeutics lead to cell death through ER stress-induced apoptosis $[29,30]$. Glioma cells are in a condition of constant lowgrade ER stress, which possibly contributes to their resistance to treatment protocols [27]. Therefore, the ER stress response could be a possible target against which to develop chemotherapeutic agents to induce toxicity in glioma cells [27]. Hence, in the current study, we investigated whether xanthatin can modify ER stress and the UPR signaling pathway in glioma cells and xenografts, leading to cell apoptosis and tumor growth blockade.

\section{MATERIALS AND METHODS}

Chemicals and reagents

Xanthatin obtained from Shizhou Biology Technology Co., Ltd. (Nanjing, China, SZSW20180145CET), was dissolved in dimethyl sulfoxide (DMSO) at a concentration of $10 \mathrm{mM}$ to make a stock solution that was stored at $-20^{\circ} \mathrm{C}$. Temozolomide (TMZ), 4-PBA (4-phenylbutyric acid), MTT (3-[4,5-dimethylthiazol-2-yl]-2,5-diphenyl-2H-tetrazolium bromide), and DAPI (4',6-diamidino-2-phenylindole hydrochloride) were purchased from Sigma (Sigma, St. Louis, MO, USA). An Annexin V/PI apoptosis detection kit was obtained from BestBio, Inc. (Shanghai, China). An enhanced 3,3'diaminobenzidine tetrahydrochloride (DAB) chromogenic kit was purchased from Zhongshan Golden Bridge Biotechnology (Beijing, China). An in situ cell death detection kit (TUNEL) was purchased from Promega (Madison, WI, USA). Serum alanine aminotransferase (ALT), aspartate aminotransferase (AST), creatinine (CRE), and urea nitrogen (BUN) reagent kits were purchased from Nanjing Jiancheng Bioengineering Institute (Nanjing, China).

\section{Cell culture and drug treatment}

The C6 rat glioma cell line and U251 human glioma cell line were obtained from the Shanghai Institute of Cell Biology, Chinese Academy of Sciences (Shanghai, China). Cells were cultured in Dulbecco's modified Eagle's medium (Invitrogen, 1710802) containing $10 \%$ fetal bovine serum, $100 \mathrm{U} / \mathrm{mL}$ penicillin, and $100 \mathrm{mg} / \mathrm{mL}$ streptomycin at $37^{\circ} \mathrm{C}$ in a $5 \% \mathrm{CO}_{2}$ humidified atmosphere. $\mathrm{C} 6$ and U251 cells were treated continuously with xanthatin at a concentration ranging from 1 to $20 \mu \mathrm{M}$ for varying periods of time ranging from 0 to $24 \mathrm{~h}$. The ER stress inhibitor 4-PBA (7 mM) was used to treat glioma cells for $4 \mathrm{~h}$ prior to xanthatin exposure.

Primary cultured glial cells were prepared from E17 embryos of pregnant Sprague-Dawley rats as described previously [31, 32]. After several days of culture, the cells were exposed to xanthatin $(1,5,10$, and $15 \mu \mathrm{M})$ for 12 or $24 \mathrm{~h}$, followed by the MTT assay.

Small interfering RNA transfection

C6 cells were transfected with 50-100 nM C/EBP-homologous protein (CHOP) siRNA using Lipofectamine 2000 reagent (Invitrogen, Carlsbad, CA, USA) according to the manufacturer's instructions. CHOP siRNA, 5'-GUCCUGUCCUCAGAUGAAATT-3' (sense) and 5'-UUUCAUCUGAGGACAGGACTT-3' (antisense), and negative control siRNA, 5'-UUCUCCGAACGUGUCACGUTT-3' (sense) and 5'ACGUGACACGUUCGGAGAATT-3' (antisense), were used for transfection (Shanghai GenePharma Co., Ltd.). Thirty-six hours after transfection, cells were treated with either $15 \mu \mathrm{M}$ xanthatin or $0.1 \%$ DMSO for another $12 \mathrm{~h}$, followed by the MTT assay or Western blotting.

MTT assay

C6 and U251 cells $\left(1 \times 10^{4}\right.$ cells/well) were seeded in 96-well plates and then exposed to xanthatin at different concentrations and exposure times. Cell viability was measured using the MTT assay as described previously [33]. The following formula was employed: cell growth inhibition rate $(\%)=(1-O D$ value of experimental group/OD value of control group) $\times 100$.

Flow cytometry with Annexin V/PI double staining

To assess cell apoptosis, C6 and U251 cells were stained with Annexin V and PI using an Annexin V-FITC/PI apoptosis detection kit according to the manufacturer's instructions and subjected to flow cytometry using a FACSVerse flow cytometer (BD Biosciences, USA).

\section{Cell cycle analysis}

The cell cycle was analyzed using flow cytometry with $\mathrm{PI}$ staining. Briefly, $12 \mathrm{~h}$ after xanthatin treatment, C6 cells $\left(2 \times 10^{6}\right)$ were harvested in triplicate. After washing twice with cold PBS, the cells were resuspended in $70 \%$ ethanol and fixed overnight at $4{ }^{\circ} \mathrm{C}$. Next, the fixed cells were washed with PBS and incubated with RNase $\mathrm{A}$ and $\mathrm{PI}$ at $37^{\circ} \mathrm{C}$ for $30 \mathrm{~min}$ in the dark. The cell cycle distribution was then analyzed with a FACSVerse flow cytometer.

\section{TUNEL staining}

Cells were exposed to xanthatin at the indicated concentrations and processed for an apoptosis assay using an in situ cell death detection kit as previously described [34]. DAPI was used to stain the nuclei. The number of TUNEL-positive cells (green) and the total number of nuclei (blue) were counted in five randomly selected fields from four different sections of each group. A histogram shows the proportion of TUNEL-positive cells in relation to the total cell number.

In vivo drug treatment of $\mathrm{C} 6$ glioma xenografts Male BALB/c athymic nude mice (4-6 weeks old, 18-20 g) were purchased from the Anhui Experimental Animal Center (Hefei, China) and used to establish a glioma xenograft model as previously described [24]. The animal surgery procedure was performed in accordance with the guideline of the Animal Care and Use Committee of Anhui Medical University. A total of $1 \times$ $10^{6} \mathrm{C} 6$ cells were resuspended in $100 \mu \mathrm{L}$ of PBS and injected subcutaneously into the right flank regions of each mouse. Once the tumor volume reached $\sim 100 \mathrm{~mm}^{3}$ at $\sim 7$ days after injection, the mice were divided randomly into five groups: a control group, xanthatin groups $(10,20$, and $40 \mathrm{mg} / \mathrm{kg})$, and a positive control TMZ group ( $5 \mathrm{mg} / \mathrm{kg}$ ) ( $n=6$ mice per group). Xanthatin dissolved in $0.5 \mathrm{~mL}$ of PBS was injected intraperitoneally into mice in the xanthatin group once per day for 2 weeks, and TMZ was intraperitoneally injected into mice in the TMZ group once per day for 1 week. Mice in the control group were injected with sterile PBS. Tumors were measured with Vernier calipers in three dimensions (Meinaite Tools, Shanghai, China), and body weights were weighed every 2 days. The tumor tissues were excised for Western blotting and immunohistochemical analysis. Blood samples were collected to measure biochemical markers of hepatic function (ALT and AST) and kidney function (BUN and CRE) using a microplate reader (Thermo Fisher Scientific, Varioskan Flash, Finland) according to the manufacturer's instructions.

Immunohistochemistry and immunofluorescence staining Tumor tissue samples were fixed in 4\% paraformaldehyde for $24 \mathrm{~h}$, dehydrated, embedded in paraffin, and sectioned at a thickness of $5 \mu \mathrm{m}$. The sections were deparaffinized in xylene and rehydrated with graded alcohol. Antigens were retrieved, and the endogenous peroxidase activity was quenched with a peroxidase suppressor. Next, the sections were blocked with $5 \%$ goat serum for $1 \mathrm{~h}$ and incubated with the corresponding primary antibodies at $4{ }^{\circ} \mathrm{C}$ overnight. Then, the appropriate biotinylated secondary antibodies were incubated with the sections for $1 \mathrm{~h}$ at $37^{\circ} \mathrm{C}$, followed by incubation with horseradish peroxidase-conjugated 
Table 1. qPCR primer details

\begin{tabular}{lll}
\hline Gene & Forward & Reverse \\
\hline GRP78 & $5^{\prime}$-GCACAGACGGGTCATTCCAC-3' & $5^{\prime}$-TCCTATGTCGCCTTCACTCC-3' \\
CHOP & $5^{\prime}$-TCTTCCTCCTCTTCCTCCTG-3' 15 & $5^{\prime}$-CACTCTTGACCCTGCTTCTC-3' \\
ATF4 & $5^{\prime}$-GCTGAGTCCGCAGCAGGTGCA-3' & $5^{\prime}$-CCCAGCTCCGGAACGAGGTCA-3' \\
PERK & $5^{\prime}$-GTCCTGTCCTCCACTCCAGA-3' & $5^{\prime}$-GGGTGTCTTCCTCCTTTATGC-3' \\
elF2a & $5^{\prime}$-GCCCACTTTCACCTTCAGAG-3' & $5^{\prime}$-CTGGTTCTTTGGTTGCTTGG-3' \\
SOD1 & $5^{\prime}$-AGGACTGCCTGGGTCTTTG-3' & $5^{\prime}$-CTTCCCGTTCATCTTCATTCA-3' \\
CAT: & $5^{\prime}$-GCAGAAGGCAAGCGGTGAAC-3' \\
GPx1 & $5^{\prime}$-GCGAATGGAGAGGCAGTGTAC-3' & $5^{\prime}$-TAGCAGGACAGCAGATGAGT-3' \\
Nrf2 & $5^{\prime}$-CTCTCCGCGGTGGCACAGT-3' \\
HIF-1a & $5^{\prime}$-GAGTGAGTTGTCTTCATTAGCACTG-3' \\
GAPDH & $5^{\prime}$-TGCCCCTGGAAGTGTCAAA-3' & $5^{\prime}$-CCACCACCGGGTCGGACATAC-3' \\
\hline
\end{tabular}

streptavidin for $15 \mathrm{~min}$ at $37^{\circ} \mathrm{C}$. Immunohistochemical staining was developed with the application of 3,3'-diaminobenzidine tetrahydrochloride $(D A B)$ for $\sim 1-3 \mathrm{~min}$. Then, the nuclei were counterstained with hematoxylin, dehydrated in graded ethanol, cleared in xylene, and observed under light microscopy. Similarly, C6 cells were grown on glass coverslips and treated with $15 \mu \mathrm{M}$ xanthatin, followed by immunohistochemistry staining using anti4-HNE antibody (1:300, Abcam, AB46545) and anti-iNOS antibody (1:400, BIOSS, bs-2072R).

C6 cells were cultured with or without xanthatin for $12 \mathrm{~h}$, and immunofluorescence staining was then performed as described previously [33]. Nuclei were stained with DAPI. Images were acquired on a Zeiss 810 confocal scanning microscope (Carl Zeiss, Inc.). Alexa Fluor 568-conjugated goat anti-rabbit lgG (A11036) was obtained from Invitrogen (Carlsbad, CA, USA).

Real-time quantitative polymerase chain reaction (qRT-PCR)

Total RNA was extracted from U251 or C6 cells using TRIzol reagent (Invitrogen, 15596018) $12 \mathrm{~h}$ after treatment with the indicated concentration of xanthatin, followed by reverse transcription to synthesize cDNA. All gene transcripts were quantified by quantitative PCR with SYBR Green QPCR Master Mix (Toyobo, Osaka, Japan) and an ABI 7500 Fast real-time PCR system (Applied Biosystems, USA). The relative expression levels of gene transcripts were evaluated using the $2^{-\Delta \Delta \mathrm{Ct}}$ method with GAPDH as the internal control. The primers used in this study are listed in Table 1.

\section{Western blot analysis}

Protein extraction and Western blot analysis were performed as described previously $[32,34]$. C6 and U251 cells were seeded on six-well plates and treated with xanthatin at various concentrations for various periods of time. The cells were washed with icecold PBS and directly extracted into lysis buffer. After electrophoresis and transfer, the PVDF membranes were blocked with $5 \%$ nonfat milk powder in TBST buffer and then incubated with primary antibodies against the following proteins at $4{ }^{\circ} \mathrm{C}$ overnight: glucose-regulated protein 78 (GRP78) (1:1000, Proteintech, 11587-1-AP), CHOP (1:1000, Santa Cruz Biotechnology, sc-793), phospho-elF2a (1:1000, CST, 3398 s), phospho-IRE1 a (1:1000, Bioss, bs-4308R), X-box binding protein-1 (XBP1s) (1:1000, BioLegend, 619502), activating factor 4 (ATF4) $(1: 1000$, CST, $11815 \mathrm{~s}$ ), activating transcription factor 6 (ATF6) $(1: 1000$, Proteintech, 24169-1-AP), cleaved caspase-3 (1:1000, CST, 9654 s), Bcl-2 (1:1000, Bioss, bs-0032R), Bax (1:500, Immunoway, YT0456), tubulin (1:1000, Sigma, t6199), and GAPDH (1:1000, BioLegend, 619502). The membranes were incubated with the corresponding horseradish peroxidase-conjugated secondary IgG for $1 \mathrm{~h}$. Blots were developed using an enhanced chemiluminescence kit (Amersham Biosciences, NJ, USA). Specific bands were evaluated by apparent molecular size. Finally, images were captured using a Bioshine ChemiQ 4600 instrument (Shanghai, China), and the intensities of the selected bands were analyzed using ImageJ software.

Statistical analysis

Quantitative data are expressed as the means \pm SEMs. Statistical comparisons were analyzed using one-way ANOVA followed by Dunnett's test. $P<0.05$ indicates a significant difference.

\section{RESULTS}

Xanthatin inhibits glioma cell viability without affecting the cell cycle

To determine the effect of xanthatin on glioma cell survival, cell viability was assessed by the MTT assay. Treatment with xanthatin for 12 and $24 \mathrm{~h}$ resulted in a higher glioma cell growth inhibition rate in a dose-dependent manner (Fig. 1a, b). However, xanthatin had little effect on the viability of primary cultured rat glial cells, especially after $12 \mathrm{~h}$ of exposure (Fig. 1c). To further investigate whether xanthatin inhibits glioma cell proliferation via inducing cell cycle arrest, flow cytometry was used to measure the cell cycle distribution of $\mathrm{C} 6$ cells treated with xanthatin. Xanthatin had no apparent effect on the percentage of cells in $G_{0} / G_{1}$ phase, $S$ phase, and $G_{2} / M$ phase (Fig. 1d, e). These results demonstrate that xanthatin decreases glioma cell proliferation but has no effect on the percentage of cells within each phase of the cell cycle.

Xanthatin promotes glioma cell apoptosis

To further investigate whether xanthatin decreases cell survival by inducing apoptosis, we performed Annexin V/PI double staining and flow cytometry in C6 and U251 glioma cells. As shown in Fig. 2a, b, xanthatin elevated the number of C6 cells in early-stage apoptosis, and $\sim 43.4 \%$ of cells underwent apoptosis (early apoptosis and late apoptosis) after treatment with $20 \mu \mathrm{M}$ xanthatin for $12 \mathrm{~h}$. TUNEL assays further revealed that xanthatin at doses of 15 and $20 \mu \mathrm{M}$ increased the number of TUNEL-positive cells compared to that in the vehicle control group (Fig. 2c, d). Consistently, xanthatin treatment induced cell apoptosis in a doseand time-dependent manner, as evidenced by increased cleaved caspase- 3 levels and decreased Bcl-2/Bax ratios in C6 (Fig. 2e, f) and U251 cells (Fig. $2 \mathrm{~g}, \mathrm{~h}$ ). These results suggest that xanthatin promotes glioma cell apoptosis. 
a

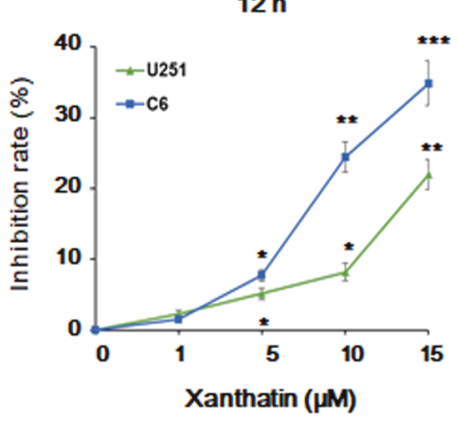

b

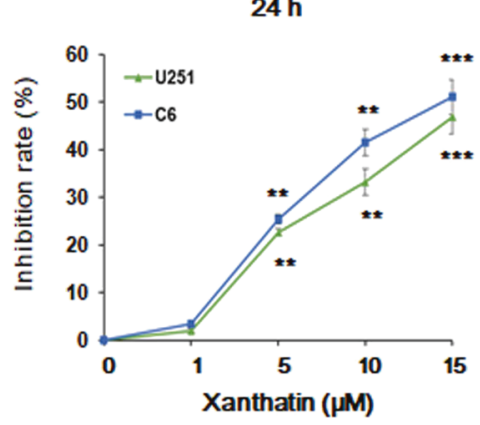

C

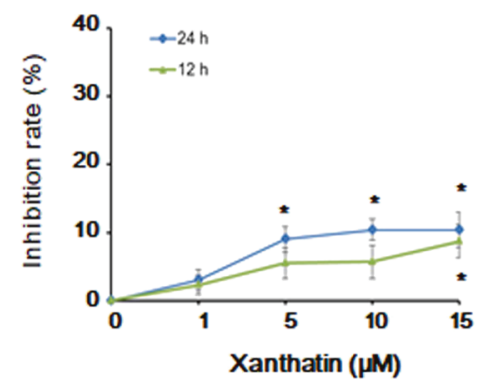

d

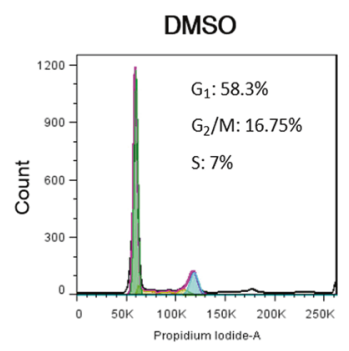

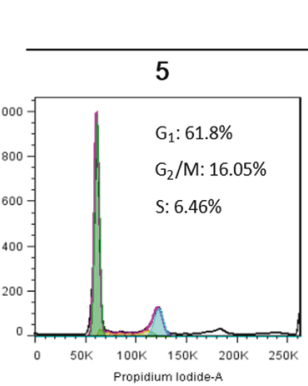

Xanthatin $(\mu \mathrm{M})$

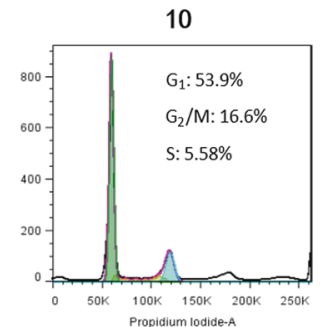

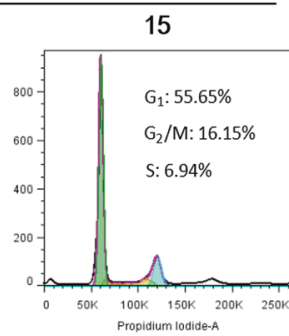

e

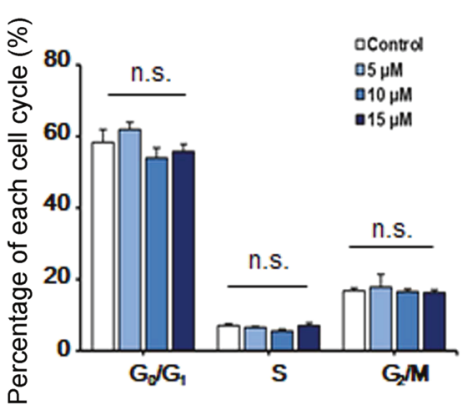

Fig. 1 Xanthatin inhibits glioma cell viability without affecting the cell cycle. The effect of xanthatin on cell viability in glioma cells. U251 and C 6 cells were incubated with xanthatin at the indicated concentrations for $12 \mathrm{~h}$ (a) or $24 \mathrm{~h}$ (b). Cell viability was determined by MTT assay. c The effect of xanthatin on cell viability in nonmalignant glial cells. Primary cultured rat glial cells were incubated with xanthatin at various concentrations for 12 or $24 \mathrm{~h}$, followed by the MTT assay. $\mathbf{d}$ Xanthatin treatment had no apparent effect on the cell cycle distribution of glioma cells. $\mathrm{C} 6$ cells were incubated with xanthatin at the indicated concentrations for $12 \mathrm{~h}$. Propidium iodide staining and flow cytometry were used to determine the proportion of cells in various phases of the cell cycle. e Statistical analysis of the cell cycle distribution in d. Statistical values are expressed as the mean \pm SEM of three independent experiments. ${ }^{*} P<0.05,{ }^{* *} P<0.01,{ }^{* *} P<0.001$ vs. the vehicle control group; n.s. no significance

Xanthatin induces ER stress and CHOP nuclear translocation in glioma cells

To determine whether xanthatin-induced glioma cell apoptosis is related to UPR activation, we examined the expression of UPR pathway-related proteins in glioma cells. A significant increase in the common ER stress marker GRP78 in C6 and U251 cells after their exposure to xanthatin indicated the occurrence of ER stress (Fig. 3). To determine whether the three canonical signaling branches of the UPR are activated in response to xanthatin treatment, the levels of phosphorylated IRE 1a (p-IRE1a) and phosphorylated elF2a (p-eIF2a), which can be phosphorylated by the phosphorylated protein kinase $R$ (PKR)-like endoplasmic reticulum kinase (PERK) kinase, were first detected. The relative levels of ( $p$-IRE1a) increased in C6 and U251 cells after xanthatin exposure compared with those in control cells (Fig. 3). Moreover, XBP1s, a key downstream signaling molecule of IRE1 that acts in the IRE1-XBP1 pathway of the UPR, was also increased in glioma cells exposed to xanthatin (Fig. 3). The levels of phosphorylated elF2a were also increased in C6 (Fig. 3a, b) and U251 cells (Fig. 3d, e) with a concomitant increase in ATF4, a transcription factor induced by the PERK-elF2a signaling pathway and increased to activate CHOP (Fig. 3). The consensus of these findings is that xanthatin $(10$ and $15 \mu \mathrm{M})$ treatment causes the nuclear translocation of CHOP, a nuclear transcription factor normally sequestered in the cytoplasm (Fig. 4b). Furthermore, xanthatin upregulated ATF6 expression, which may lead to a further increase in CHOP (Fig. 3). Real-time quantitative polymerase chain reaction ( $\mathrm{QRT}-\mathrm{PCR}$ ) also demonstrated that xanthatin upregulated the levels of the UPR-related genes GRP78, XBP1s, ATF4, CHOP, and PERK but had no apparent effect on elF2a levels (Fig. 4a). Collectively, these findings indicate that xanthatin induces ER stress concomitant with CHOP activation in glioma cells.

Xanthatin suppresses the growth of glioma in BALB/c athymic nude mice and induces ER stress-related apoptosis

To evaluate the in vivo anticancer effect of xanthatin, athymic mice bearing glioma xenografts received the intraperitoneal (i.p.) injection of xanthatin for 14 consecutive days. Xanthatin decreased the volume of glioma tumors in a dose-dependent manner compared with those in the vehicle control group. TMZ, an alkylating agent widely used to treat primary and recurrent high-grade gliomas, also decreased the tumor size to a level similar to that of the group exposed to $20 \mathrm{mg} / \mathrm{kg}$ xanthatin (the middle dose) (Fig. 5b). When the tumors were removed, the average weight of the tumors from mice treated with xanthatin at the $40 \mathrm{mg} / \mathrm{kg}$ dose was twofold lower than that of tumors from the vehicle-treated mice (Fig. $5 \mathrm{c}$ ). Moreover, necrotic areas in the tumor tissues of glioma xenograft mice injected with xanthatin as well as mice in the TMZ group rapidly increased (Supplementary Fig. S1b). Furthermore, xanthatin, especially at $40 \mathrm{mg} / \mathrm{kg}$, significantly upregulated the ER stress proteins phospho-IRE1, ATF6, phospho-eIF2a, XBP1s, and ATF4 (Fig. 5d, e), which is consistent with the in vitro results. The increased immunoreactivity of GRP78 and CHOP in tumor tissues was also observed in xanthatin-treated xenograft mice (Fig. 5f). Furthermore, the expression of CHOP and cleaved caspase-3 in tumor tissues was also elevated in the xanthatin-treated groups compared to that in the control group (Fig. 5d, e). However, xanthatin had no significant effects on body weight (Fig. 5a) or the serum contents of the hepatic and renal function markers ALT, AST, BUN, and CRE (Supplementary Fig. 
a

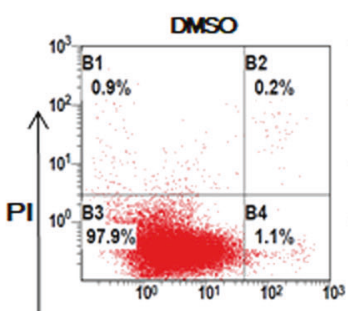

Annexin V

C
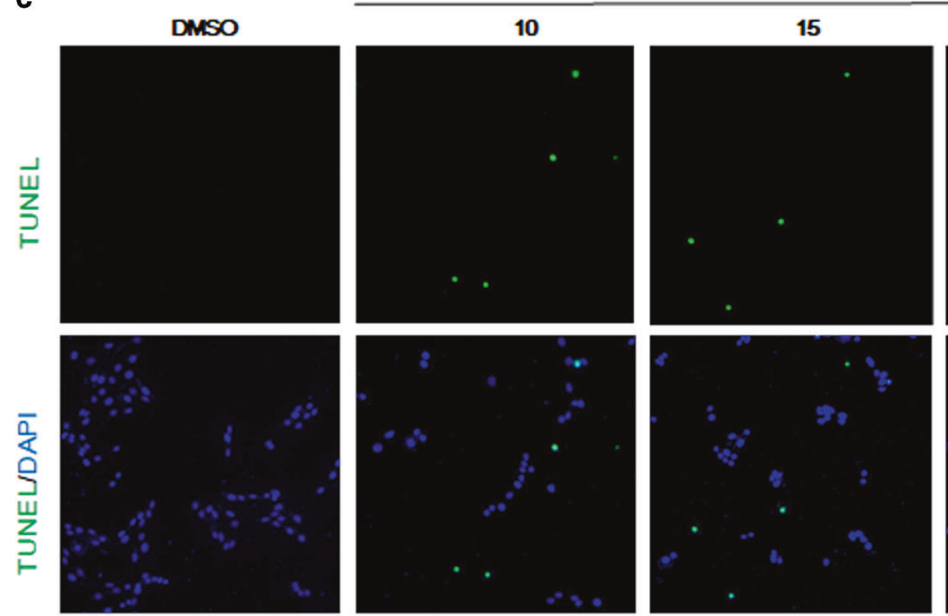

f

e
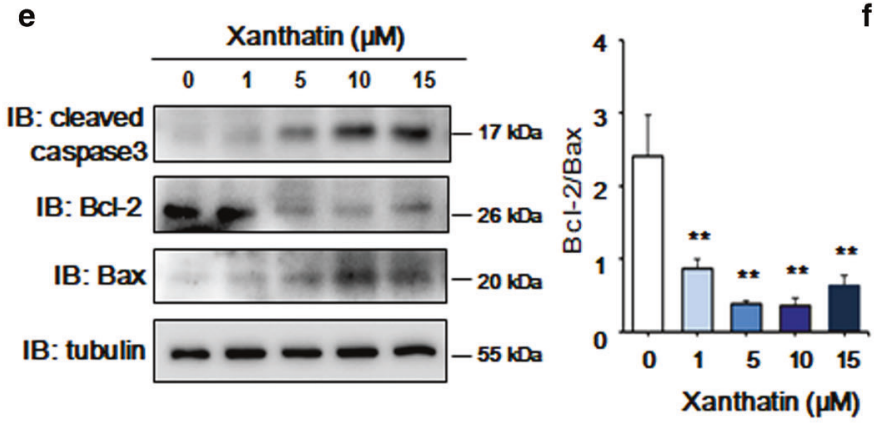

g
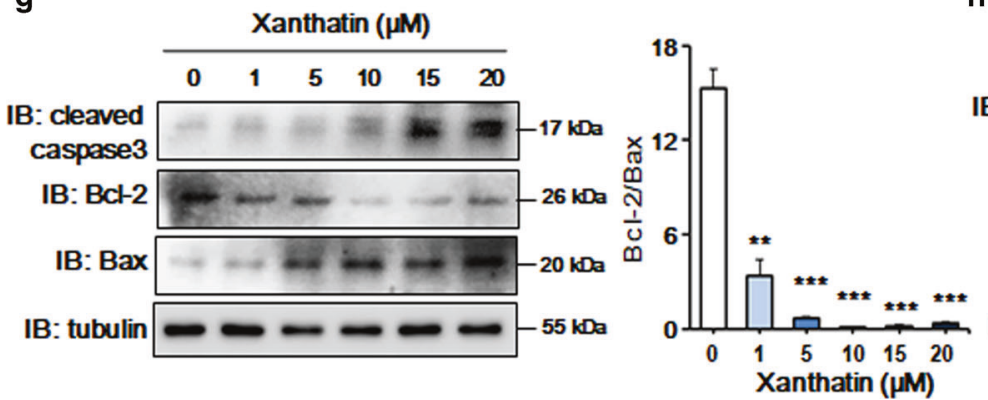

h b

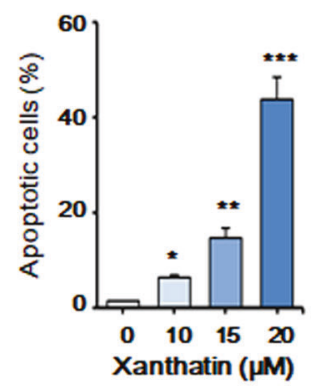

d

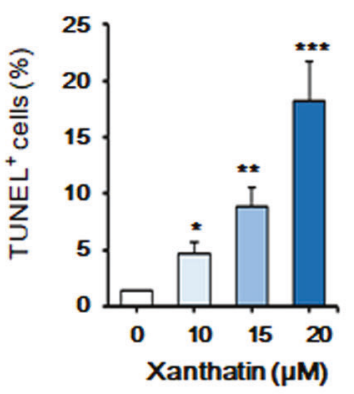

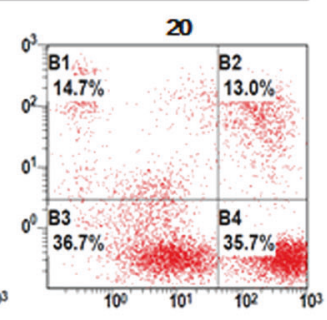

$\mathbf{2 0}$

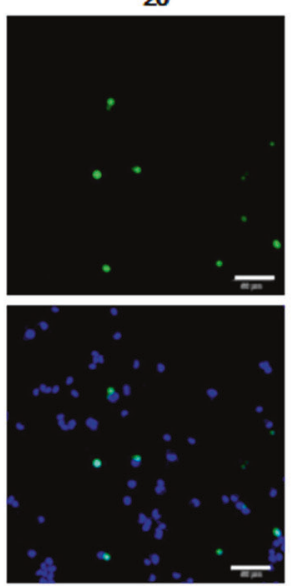

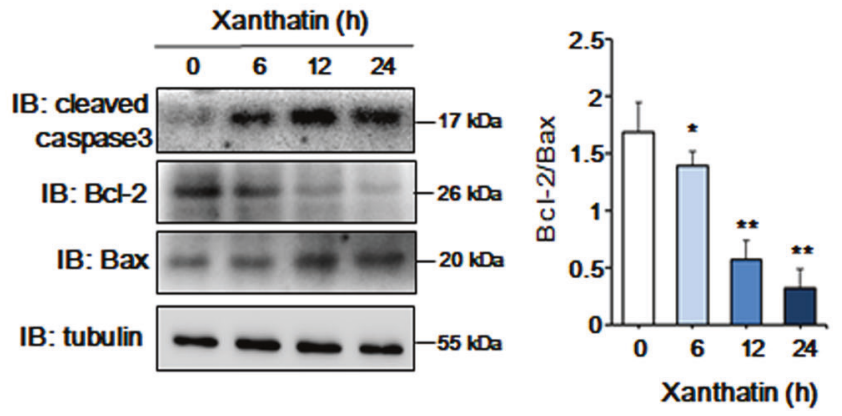
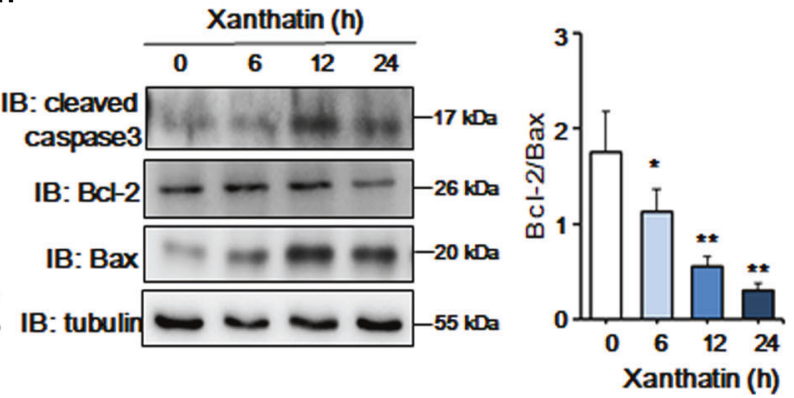

Fig. 2 Xanthatin promotes the apoptosis of glioma cells. a The apoptosis rate of glioma cells was determined by flow cytometry with Annexin V/PI staining. C6 cells were incubated with 10,15 , and $20 \mu \mathrm{M}$ xanthatin for $12 \mathrm{~h}$. DMSO was used as a vehicle control. b Quantitative analysis of apoptotic cells in a. c TUNEL staining of C6 cells treated with xanthatin at the indicated concentrations for $12 \mathrm{~h}$. $\mathbf{d}$ Quantitative analysis of TUNEL-positive C 6 cells in c. e C 6 cells were incubated with xanthatin at various concentrations for $12 \mathrm{~h}$, and the levels of cleaved caspase-3, $\mathrm{Bcl}-2$, Bax, and tubulin were detected by WB. f C 6 cells were incubated with $15 \mu \mathrm{M}$ xanthatin for 6,12 , and $24 \mathrm{~h}$, and the levels of cleaved caspase-3, Bcl-2, Bax, and tubulin were determined by WB. g U251 cells were incubated with various concentrations of xanthatin for $12 \mathrm{~h}$, and the levels of cleaved caspase-3, Bcl-2, Bax, and tubulin were detected by WB. h U251 cells were incubated with $15 \mu \mathrm{M}$ xanthatin for 6,12 , and $24 \mathrm{~h}$, and the expression levels of cleaved caspase-3, Bcl-2, Bax, and tubulin were determined by WB. Densitometric quantitative analysis of the $\mathrm{BCl}-2 / \mathrm{Bax}$ ratio, which is expressed as the mean \pm SEM of three independent experiments. ${ }^{*} P<0.05,{ }^{* *} P<0.01,{ }^{* * *} P<0.001$ vs. control Scale bar $=50 \mu \mathrm{m}$ 
a

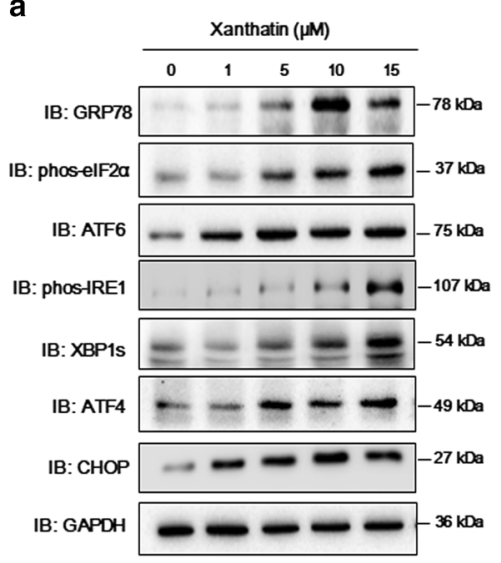

d

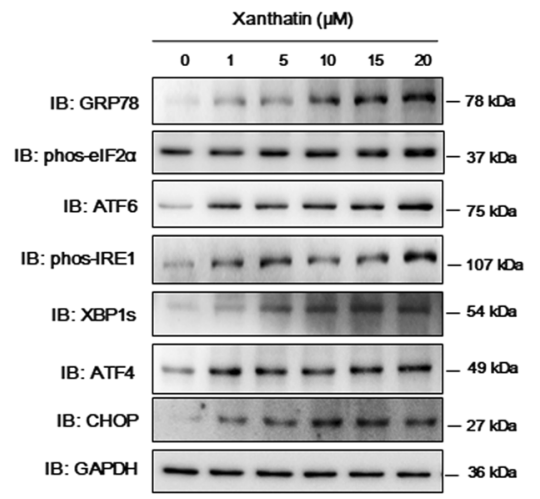

b

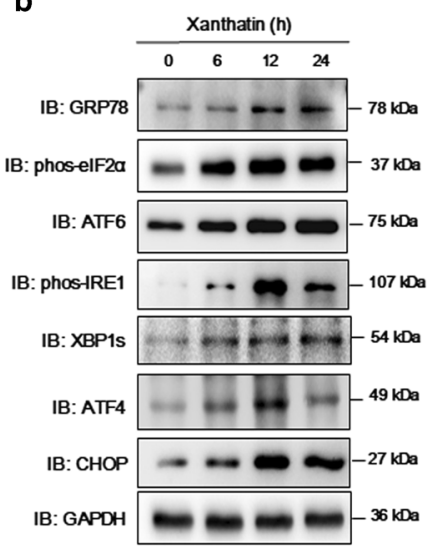

e

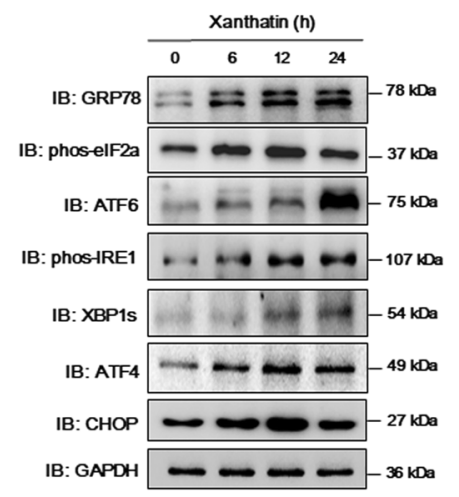

C
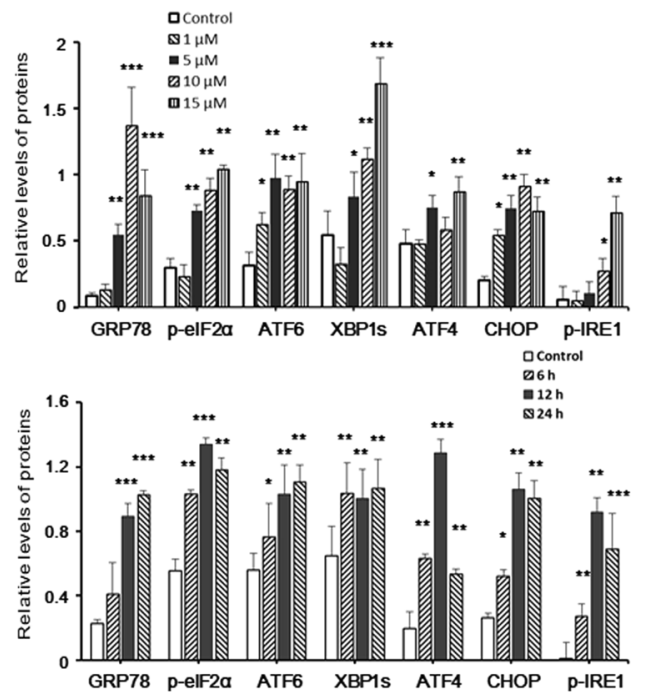

f

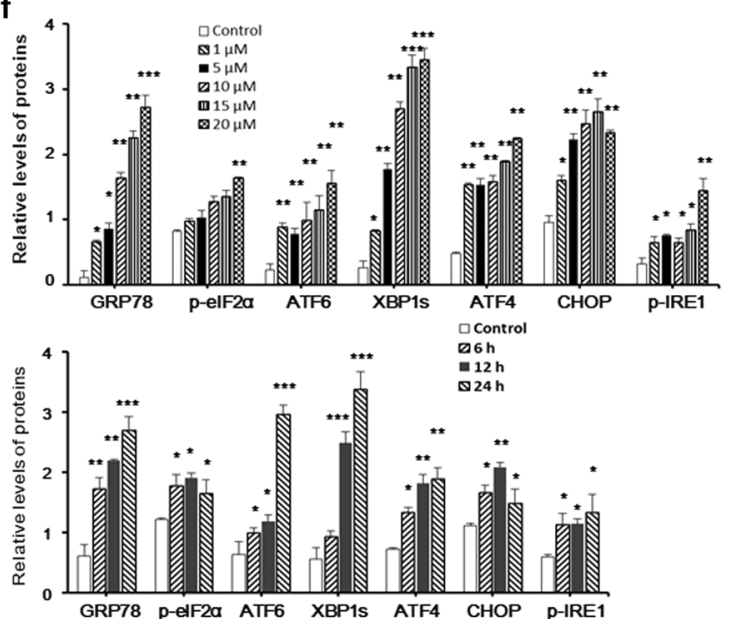

Fig. 3 Xanthatin induces ER stress in glioma cells. a Representative immunoblots against ER stress-related proteins from C6 cells treated with xanthatin $(1,5,10$, and $15 \mu \mathrm{M})$ for $12 \mathrm{~h}$. b Representative immunoblots against ER stress-related proteins from C6 cells treated with $15 \mu \mathrm{M}$ xanthatin for the indicated times. c Quantitative analysis of protein levels in a and $\mathbf{b}$. d The levels of ER stress-related proteins in U251 cells treated with xanthatin at the indicated concentrations for $12 \mathrm{~h}$. e The levels of ER stress-related proteins in U251 cells treated with xanthatin for 6,12 , and $24 \mathrm{~h}$. f Quantitative analysis of protein levels in $\mathbf{d}$ and e. Values are expressed as the mean \pm SEM of three independent experiments. ${ }^{*} P<0.05,{ }^{* *} P<0.01,{ }^{* * *} P<0.001$ vs. control

S1a). These findings indicate that xanthatin triggers ER stressrelated cell death in glioma xenografts.

The ER stress inhibitor 4-PBA and CHOP siRNA partially attenuate xanthatin-induced cytotoxicity against glioma cells

To verify the role of ER stress in xanthatin-induced cell apoptosis, the ability of 4-PBA, a chemical chaperone that inhibits ER stress through reducing the accumulation of misfolded proteins in the ER $[35,36]$, to ameliorate the cytotoxic effects of xanthatin on glioma cells was tested. Pretreatment with 4-PBA increased the viability of $\mathrm{C} 6$ cells treated with xanthatin compared with that of C6 cells treated with xanthatin only (Fig. 6a). Furthermore, 4-PBA partially reversed the increased levels of cleaved caspase- 3 and $\mathrm{CHOP}$ as well as the decreased $\mathrm{Bcl}-2 / \mathrm{Bax}$ ratio induced by xanthatin (Fig. 6b-e). As a positive control, a commercially available ER stress inducer, tunicamycin (TM), was applied to enhance ER stress. TM treatment resulted in a dramatic increase in CHOP and cleaved caspase- 3 expression and a significantly decreased $\mathrm{Bcl}-2 / \mathrm{Bax}$ ratio in $\mathrm{C} 6$ cells, which were also partially attenuated by pretreatment with 4-PBA (Fig. 6b-e). Meanwhile, the TUNEL assay showed the relatively decreased proportion of
TUNEL-positive C6 cells pretreated with 4-PBA for $4 \mathrm{~h}$ and xanthatin for $12 \mathrm{~h}$ compared with that in $\mathrm{C} 6$ cells treated with only xanthatin (Fig. $6 f, g$ ). These results suggest that ER stress is involved in xanthatin-induced glioma cell death.

To further explore whether the proapoptotic factor CHOP is a vital contributor to xanthatin-induced cell apoptosis, CHOP siRNA was transfected into $\mathrm{C} 6$ cells. As shown in Fig. 6, C6 cell growth was increased by $36.5 \%$ with xanthatin exposure concomitant with CHOP knockdown compared with the growth of xanthatinand NC-siRNA-treated cells (Fig. 6h). Further investigation showed that the knockdown of CHOP decreased cleaved caspase-3 expression and increased the $\mathrm{BCl}-2 / \mathrm{Bax}$ ratio in xanthatin-treated cells compared with those in NC-siRNA transfected cells (Fig. 6i-k). Taken together, these results suggest that xanthatin-induced glioma cell apoptosis is at least partially mediated by the ER stress pathway involving CHOP induction.

\section{DISCUSSION}

Although xanthatin has been implicated as an antitumor therapy in a wide variety of human cancers in vitro $[6,37,38]$, no detailed 
a
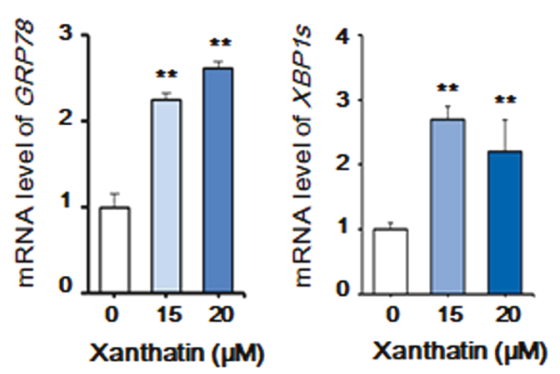

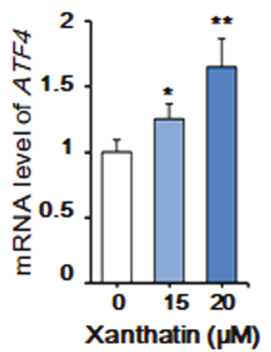

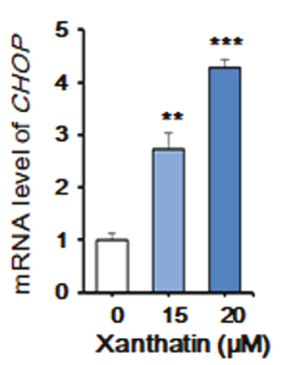
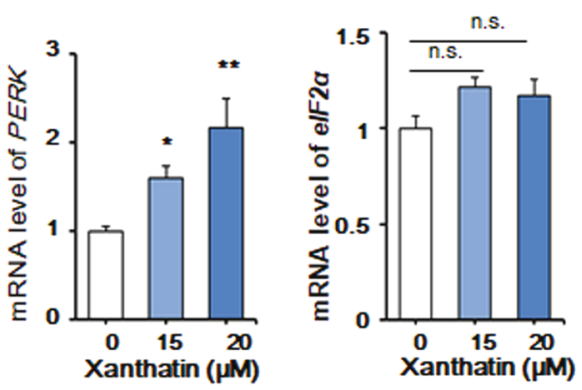

b

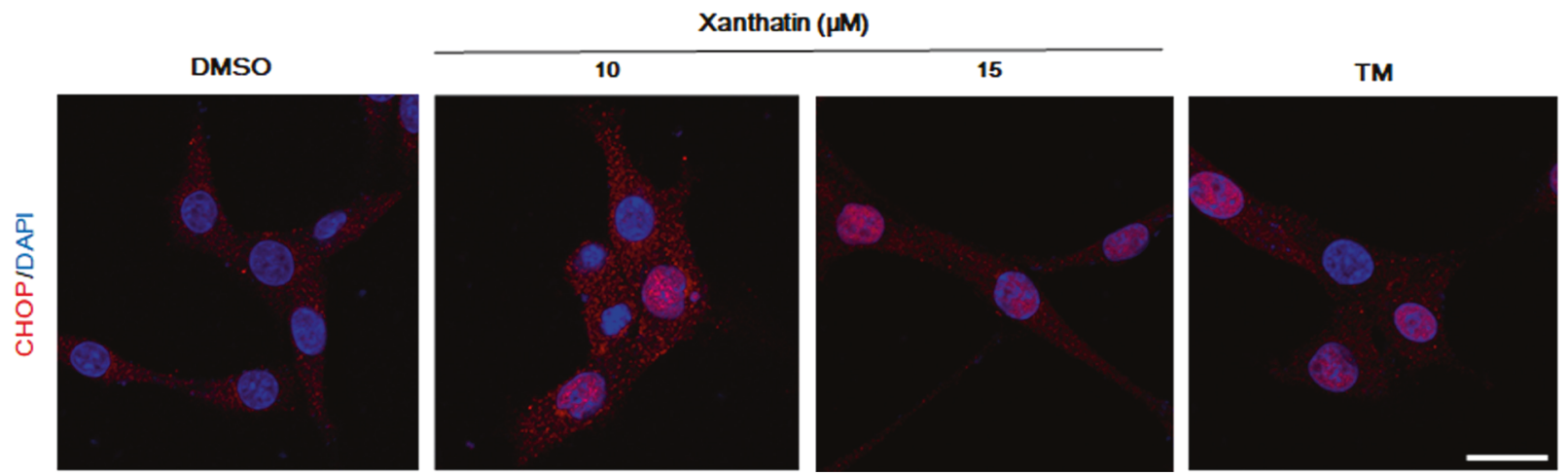

Fig. 4 Xanthatin upregulates the expression of ER stress-related genes and induces the nuclear translocation of CHOP in glioma cells. a U251 cells were treated with xanthatin $(15$ and $20 \mu \mathrm{M})$ for $12 \mathrm{~h}$, and the mRNA levels of GRP78, XBP1s, ATF4, CHOP, PERK, and elF2a were assessed by qRT-PCR. GAPDH was used as a control. Values are expressed as the mean \pm SEM of three independent experiments. ${ }^{*} P<$ $0.05,{ }^{* *} P<0.01,{ }^{* * *} P<0.001$ vs. the vehicle control group. n.s. no significance. b Representative images showing the nuclear translocation of $\mathrm{CHOP}$ in $\mathrm{C} 6$ cells treated with xanthatin $(10$ and $15 \mu \mathrm{M})$ or the ER stress inducer tunicamycin (TM). Scale bar $=50 \mu \mathrm{m}$

studies on its effect on glioma cell proliferation or apoptosis have been conducted. Our previous data from a radiography study showed that the antiglioma effect of xanthatin is associated with the inhibition of angiogenesis [24]. The exact molecular mechanisms of the antitumor activity of xanthatin have yet to be elucidated. In the present study, we highlighted the strong doseand time-dependent inhibitory effect of xanthatin on glioma cell growth. More importantly, xanthatin had little influence on the viability of nonmalignant glial cells. These data were further confirmed using C6 xenograft mice, which showed retarded tumor growth and reduced tumor weight following xanthatin administration. Meanwhile, xanthatin had no significant influence on body weight or liver or kidney function in xenograft mice. These results indicate that xanthatin has a relatively low cytotoxicity and may have great potential for glioma therapy in clinical applications.

Xanthatin-induced significant cell apoptosis in glioma cells, which suggests that the xanthatin-induced inhibition of glioma growth relies predominantly on the induction of apoptosis. Xanthatin has no effect on cycle arrest in glioma cells, which is in contrast to the observations of other groups who showed that xanthatin exerted its antitumor activity via inducing $G_{2} / M$ cell cycle arrest in human lung cancer cells, gastric carcinoma cells, and human breast cancer cell lines [15, 20-22]. This difference in results may be associated with the tumor types examined.

To further investigate the mechanism underlying the induction of apoptosis by xanthatin, we characterized the effect of xanthatin on signaling pathways associated with cell apoptosis. The xanthatin analogue $1 \beta$-hydroxyl-5a-chloro-8-epi-xanthatin induces apoptosis through ROS-mediated ERK/p38 MAPK activation and JAK2/STAT3 inhibition in human hepatocellular carcinoma [37]. In addition, xanthatin induces tumor cell apoptosis through a p53dependent intrinsic mitochondrial pathway, oxidative stress, and the inactivation of NF-KB $[6,20,22]$. Whether ER stress plays an important role in xanthatin-induced glioma cell apoptosis remains obscure. Increasing evidence has shown that ER stress is a doubleedged sword that acts as a cell death mechanism and also prolongs the survival of cancer cells in tumorigenesis $[25,29,30,39]$. Thus, numerous current anticancer therapies to induce ER stress to stimulate its proapoptotic function or block its prosurvival function have been devised $[3,27,39]$. The PERKelF2a-ATF4-CHOP pathway is one of the major UPR pathways [25]. PERK signaling activation is largely sustained with unmitigated ER stress, and sustained PERK signaling impairs cell proliferation and promotes apoptosis [40]. By phosphorylating elF2 $\alpha$ and activating ATF4, PERK induces CHOP expression [41]. CHOP downregulates $\mathrm{Bcl}-2$ expression and increases the translocation of Bax from the cytosol to mitochondria, which consequently triggers the activation of caspase-3, resulting in cell apoptosis [25]. In our study, following xanthatin exposure, the levels of phospho-elF2a, ATF4, and CHOP were dramatically increased in glioma cells and xenografts, accompanied by the nuclear translocation of $\mathrm{CHOP}$, suggesting that the PERK-eIF2a-ATF4-CHOP pathway is involved in xanthatin-induced cell apoptosis. CHOP knockdown afforded C6 cells increased resistance to xanthatin-induced apoptosis, further confirming that CHOP is a central factor involved in xanthatininduced glioma cell apoptosis.

Xanthatin also increased phospho-IRE1a, spliced XBP1, and ATF6 levels, which implied that these two UPR signaling pathways are also involved in xanthatin-induced glioma cell apoptosis. The ER stress inhibitor 4-PBA partially ameliorated xanthatin-induced tumor cell growth inhibition and decreased the apoptotic rate in glioma cells, which also confirms the key role of ER stress in xanthatin activity. In view of the limited number of indicators used in this study, we cannot exclude the possibility that other UPRrelated proteins or apoptosis-associated signaling pathways are 
a

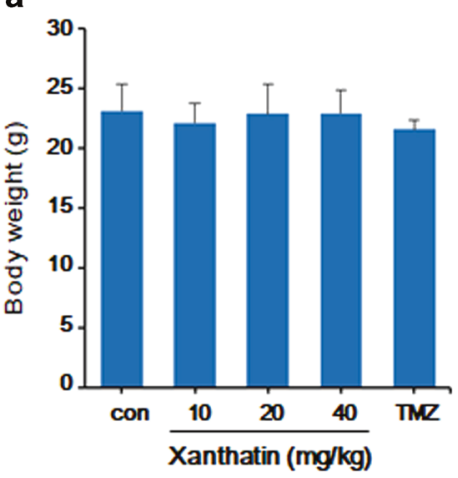

b

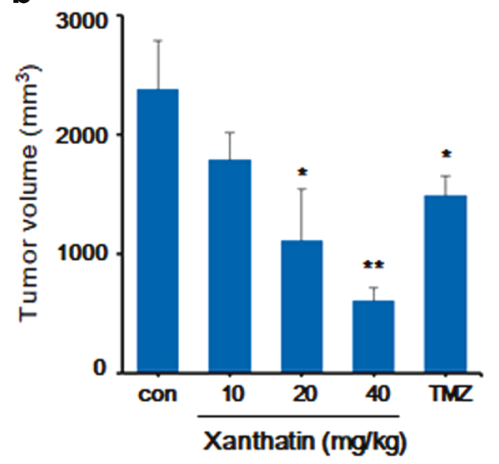

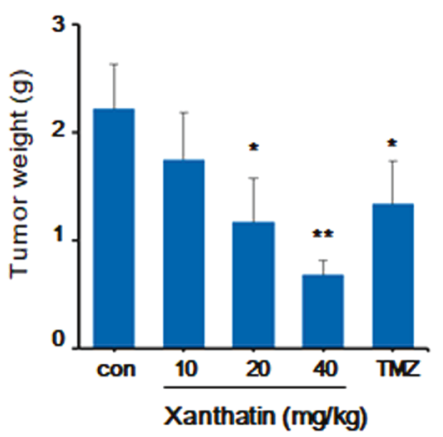

d

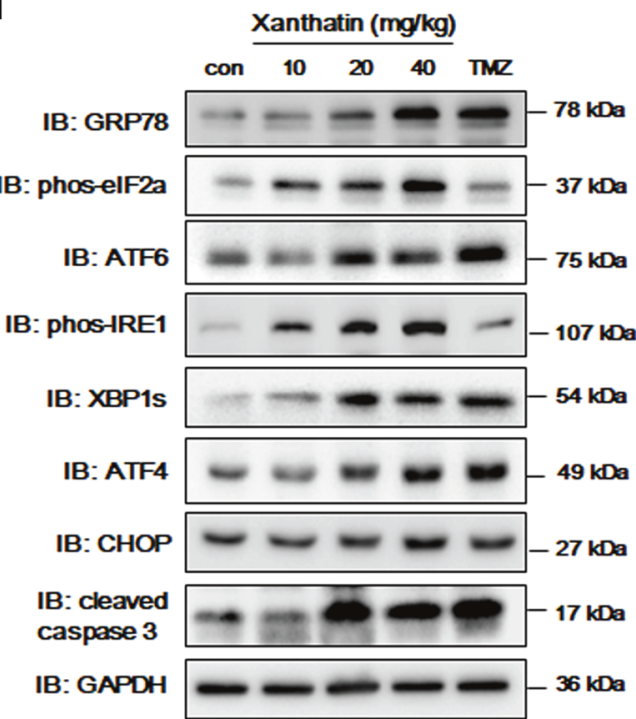

e

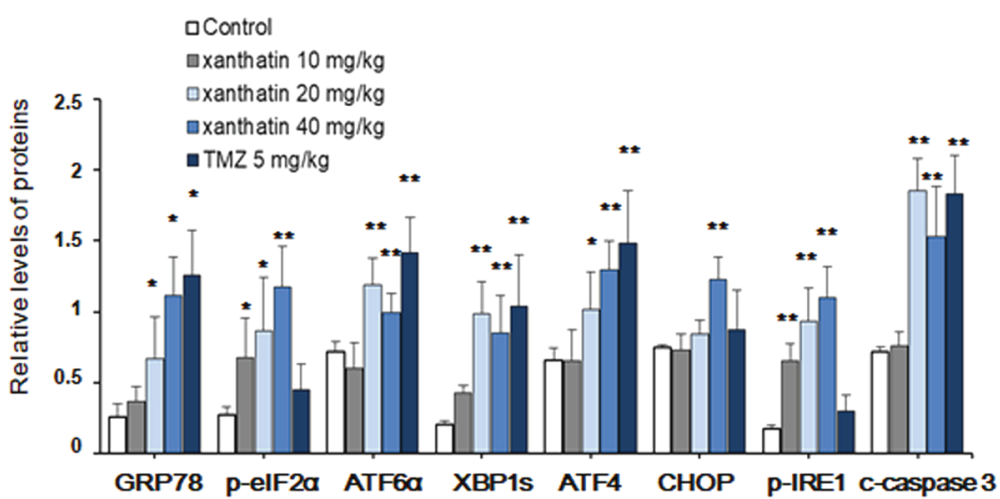

f

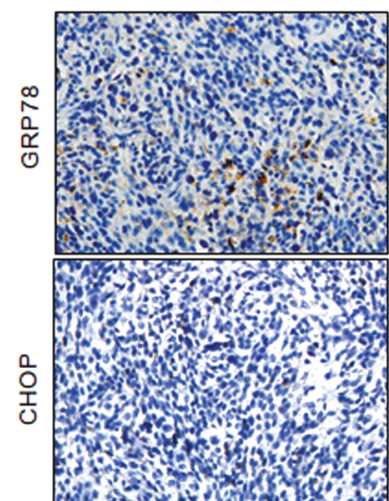

\section{Xanthatin $(\mathrm{mg} / \mathrm{kg})$}
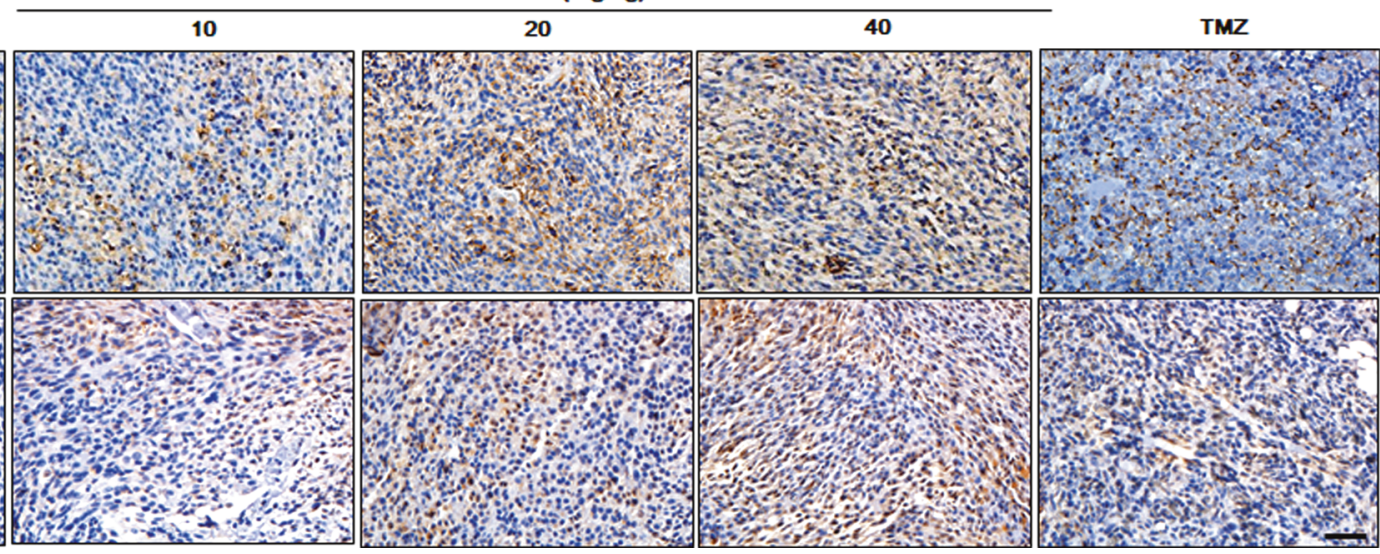

Fig. 5 Xanthatin inhibits glioma growth and induces ER stress in athymic BALB/c nude mice. The inhibitory effect of xanthatin on glioma growth. A total of $1 \times 10^{6} \mathrm{C} 6$ cells were injected subcutaneously into the right flank regions of each nude mouse. Different concentrations of xanthatin or temozolomide (TMZ) were intraperitoneally (i.p.) injected once per day. Body weights (a), tumor volumes (b), and tumor weights (c) were measured on the 13th day of continuous xanthatin administration or the 6th day of TMZ injection. $n=5$ mice per group. Values are expressed as the mean \pm SEM. ${ }^{*} P<0.05,{ }^{* *} P<0.01$ vs. the vehicle group. $\mathbf{d}$ Immunoblots against ER stress-related proteins and cleaved caspase3 in xenograft tumors. e Quantitative analysis of protein levels in d. $n=5$ in each group. Values are expressed as the mean \pm SEM of three independent experiments. ${ }^{*} P<0.05,{ }^{* * *} P<0.01$ vs. the vehicle group $c$-caspase- 3 cleaved caspase-3. $\mathbf{f}$ Representative immunohistochemical staining for GRP78 and CHOP in five groups of mice that received xanthatin or TMZ at different concentrations after tumor implantation. Scale bar $=50 \mu \mathrm{m}$ 

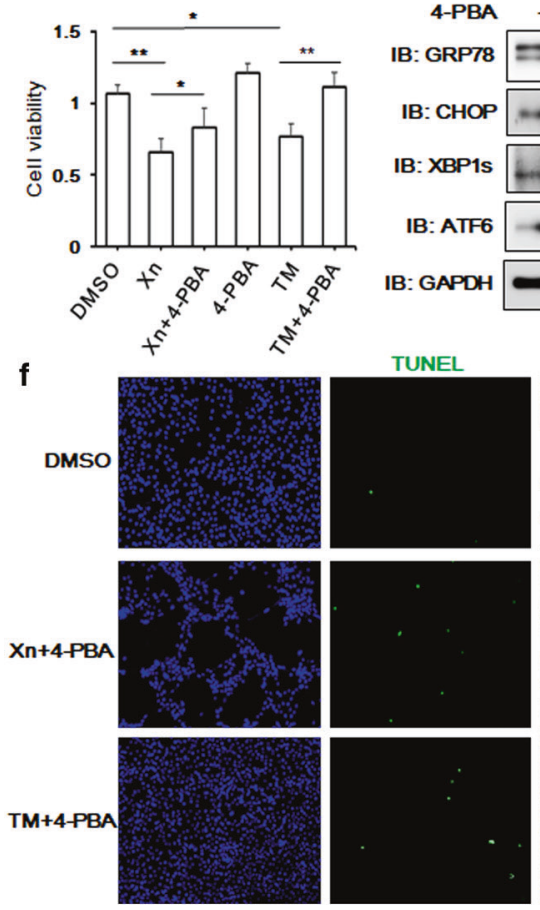

h

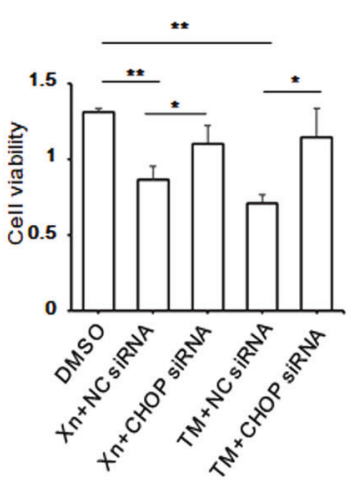

i DMSO b

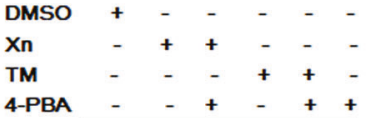

IB: GRP78 $=\geq=2=-78 \mathrm{kDa}$

IB: $\mathrm{CHOP} \cdots \omega \omega \omega-27 \mathrm{kDa}$

IB: XBP1s

IB: ATF6

IB: GAPDH
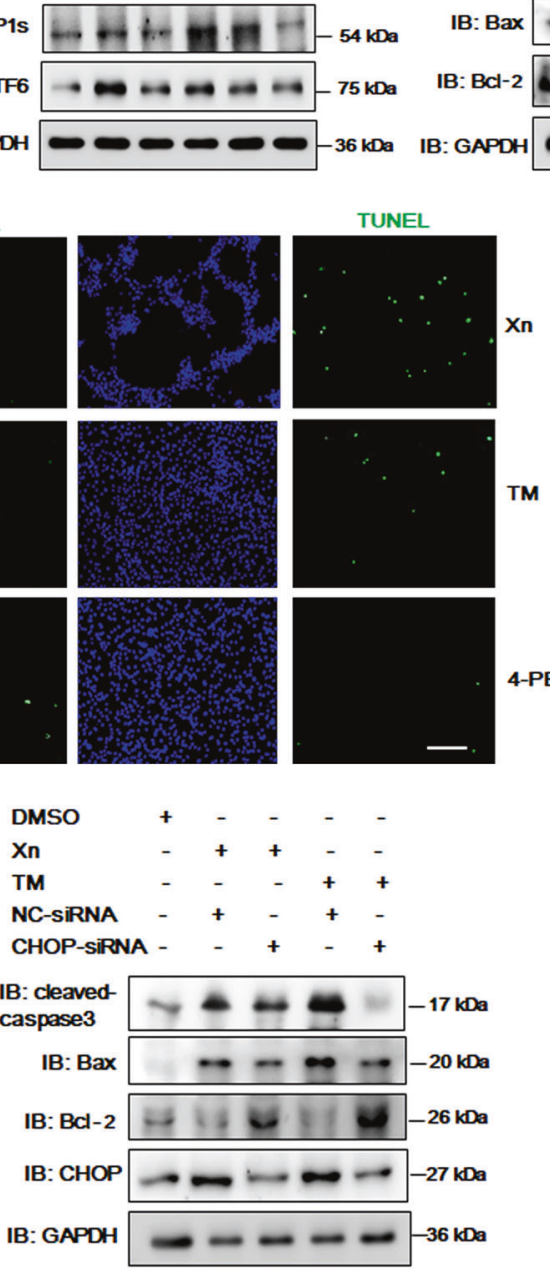

g

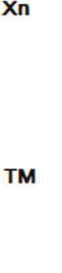

C

$\begin{array}{lllllll}\text { DMSO } & + & - & - & - & - & - \\ X_{n} & - & + & + & - & - & -\end{array}$

4-PBA - -++-+

TM

B: cleaved-

IB: Bax

IB: Bcl-2
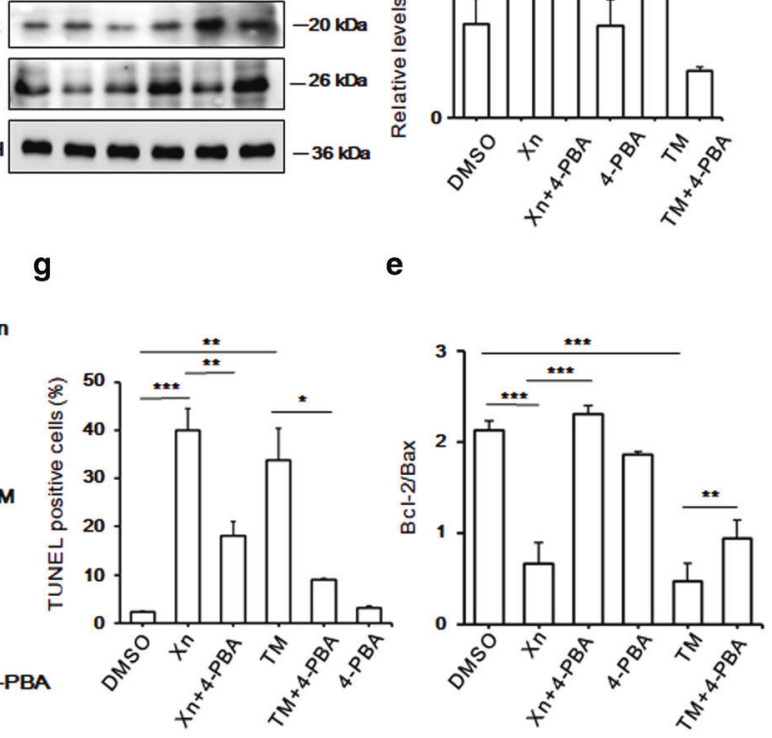

j

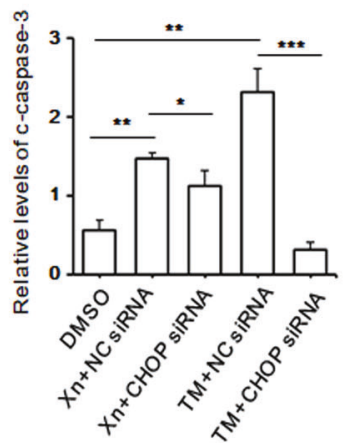

d

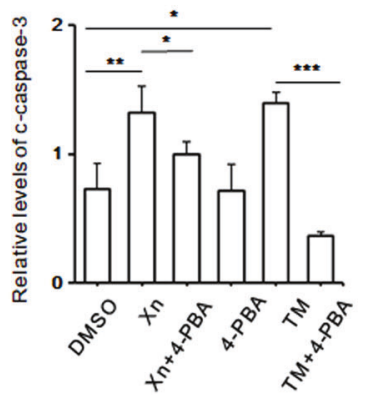

e

k

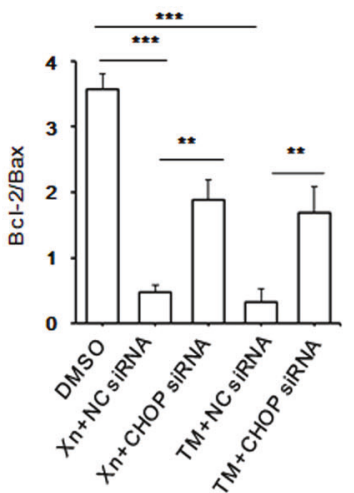

Fig. 6 ER stress, especially CHOP, is involved in the xanthatin-induced apoptosis of glioma cells. C6 cells were pretreated with the ER stress inhibitor 4-PBA $(7 \mathrm{mM})$ for $4 \mathrm{~h}$, followed by incubation with xanthatin $(15 \mu \mathrm{M})$ or TM $(2.5 \mu \mathrm{M})$ for $12 \mathrm{~h}$. a The MTT assay was used to detect cell viability. $\mathbf{b}$ The expression levels of the ER stress-related proteins GRP78, CHOP, XBP1s, and ATF6 were determined by WB. c The protein levels of cleaved caspase-3, Bcl-2, and Bax in C6 cells. d Quantitative analysis of cleaved caspase-3 levels in c. e Quantitative analysis of the Bcl-2/Bax ratio in c. f TUNEL staining showing apoptotic cells in C6 cells following the indicated treatment. Scale bar $=100 \mu \mathrm{m}$. $\mathbf{g}$ Quantitative analysis of TUNEL-positive cells in $\mathbf{f}$. $\mathbf{h}$ C6 cells were transfected with NC-siRNA or CHOP siRNA for 36 , followed by incubation with $15 \mu M$ xanthatin for another $12 \mathrm{~h}$. Cell viability was determined by the MTT assay. i The protein levels of cleaved caspase-3, Bcl-2, Bax, and CHOP in C6 cells transfected with CHOP siRNA. j Quantitative analysis of cleaved caspase-3 levels in i. k Quantitative analysis of the Bcl-2/Bax ratio in i. Values are expressed as the mean \pm SEM of three independent experiments. ${ }^{*} P<0.05,{ }^{* * *} P<0.01,{ }^{* * *} P<0.001$. Xn xanthatin, TM tunicamycin, c-caspase3 cleaved caspase- 3

involved in xanthatin-induced cell death. Although our preliminary data showed that xanthatin has no effect on the expression of five oxidative stress-related genes in C6 cells (CAT, SOD1, GPX1, NRF2, and HIF-1a) (Supplementary Fig. S2a), oxidative stress evaluated by 4-hydroxynonenal (4-HNE) staining was slightly increased in xanthatin-treated cells (Supplementary Fig. S2b). Therefore, whether oxidative stress, especially that revealed by 4$\mathrm{HNE}$, is involved in xanthatin-induced glioma cell apoptosis and the underlying mechanisms of the effects of xanthatin require further study.

\section{CONCLUSION}

In summary, as illustrated in Fig. 7, our current study highlights that xanthatin treatment potentiates ER stress in glioma cell lines and xenograft tumors, resulting in cell apoptosis and tumor growth inhibition. Treatment with the classic ER stress inhibitor 4PBA or CHOP knockdown partially ameliorated the cytotoxic effects of xanthatin on glioma cells concomitant with the downregulation of cleaved caspase-3. These results may be useful in identifying xanthatin as a potential drug candidate for the targeted therapeutic intervention of glioma. 


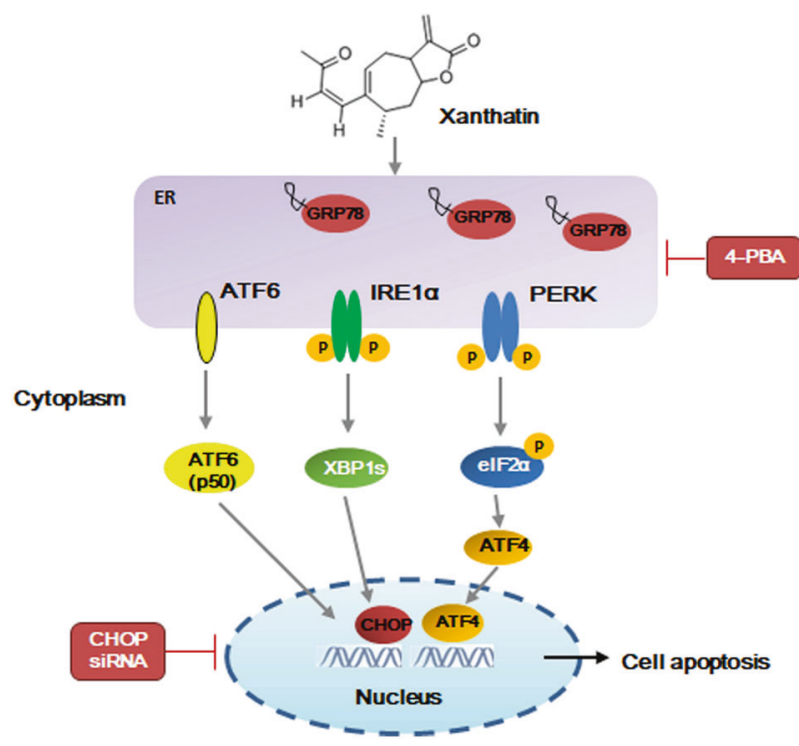

Fig. 7 Schematic diagram of xanthatin-induced ER stress-related apoptosis in glioma cells. Xanthatin triggers three proximal ER stress sensors, PERK, IRE1, and ATF6, leading to CHOP induction and nuclear translocation, which further regulates the expression of apoptosis-related genes and triggers glioma cell death. Treatment with the ER stress inhibitor 4-PBA or CHOP knockdown partially ameliorates the antitumor effects of xanthatin

\section{ACKNOWLEDGEMENTS}

This study was financially supported by the National Natural Science Foundation of China (81673438 to YXS, 81302755 and 81973337 to LJF), the State's Key Project of Research and Development Plan (YFC1305902 to YXS), the Key Projects of Anhui Provincial Natural Science Research in Colleges and Universities (KJ2017A167 to LJF), and the Scientific Research Foundation of the Institute for Translational Medicine of Anhui Province (2017zhyx34 to YXS and YQY).

\section{AUTHOR CONTRIBUTIONS}

LJF, YXS, and YQY designed the research; YYM, ZMD, QC, WSX and SXB performed the research; YYM, ZMD, JSY, and YJS analyzed the data; and LJF and YXS wrote the paper.

\section{ADDITIONAL INFORMATION}

The online version of this article (https://doi.org/10.1038/s41401-019-0318-5) contains supplementary material, which is available to authorized users.

Competing interests: The authors declare no competing interests.

\section{REFERENCES}

1. Szopa W, Burley TA, Kramer-Marek G, Kaspera W. Diagnostic and therapeutic biomarkers in glioblastoma: current status and future perspectives. Biomed Res Int. 2017;2017:8013575.

2. Lapointe S, Perry A, Butowski NA. Primary brain tumours in adults. Lancet. 2018;392:432-46.

3. Ghotme KA, Barreto GE, Echeverria V, Gonzalez J, Bustos RH, Sanchez M, et al. Gliomas: new perspectives in diagnosis, treatment and prognosis. Curr Top Med Chem. 2017;17:1438-47.

4. Lee BH, Yoon SH, Kim YS, Kim SK, Moon BJ, Bae YS. Apoptotic cell death through inhibition of protein kinase CKII activity by 3,4-dihydroxybenzaldehyde purified from Xanthium strumarium. Nat Prod Res. 2008:22:1441-50.

5. Aranjani JM, Manuel A, Mallikarjuna Rao C, Udupa N, Rao JV, Joy AM, et al. Preliminary evaluation of in vitro cytotoxicity and in vivo antitumor activity of Xanthium strumarium in transplantable tumors in mice. Am J Chin Med. 2013;41:145-62.

6. Liu R, Shi D, Zhang J, Li X, Han X, Yao X, et al. Xanthatin promotes apoptosis via inhibiting thioredoxin reductase and eliciting oxidative stress. Mol Pharm. 2018;15:3285-96.
7. Ramirez-Erosa I, Huang Y, Hickie RA, Sutherland RG, Barl B. Xanthatin and xanthinosin from the burs of Xanthium strumarium L. as potential anticancer agents. Can J Physiol Pharmacol. 2007;85:1160-72.

8. Shen $M$, Zhou XZ, Ye L, Yuan Q, Shi C, Zhu PW, et al. Xanthatin inhibits corneal neovascularization by inhibiting the VEGFR2 mediated STAT3/PI3K/Akt signaling pathway. Int J Mol Med. 2018;42:769-78.

9. Yu Y, Yu J, Pei CG, Li YY, Tu P, Gao GP, et al. Xanthatin, a novel potent inhibitor of VEGFR2 signaling, inhibits angiogenesis and tumor growth in breast cancer cells. Int J Clin Exp Pathol. 2015;8:10355-64.

10. Romero M, Zanuy M, Rosell E, Cascante M, Piulats J, Font-Bardia M, et al. Optimization of xanthatin extraction from Xanthium spinosum L. and its cytotoxic, anti-angiogenesis and antiviral properties. Eur J Med Chem. 2015;90:491-6.

11. Nibret $E$, Youns $M$, Krauth-Siegel RL, Wink M. Biological activities of xanthatin from Xanthium strumarium leaves. Phytother Res. 2011;25:1883-90.

12. Pinel B, Landreau A, Seraphin D, Larcher G, Bouchara JP, Richomme P. Synthesis of reduced xanthatin derivatives and in vitro evaluation of their antifungal activity. J Enzym Inhib Med Chem. 2005;20:575-9.

13. Lavault M, Landreau A, Larcher G, Bouchara JP, Pagniez F, Le Pape P, et al. Antileishmanial and antifungal activities of xanthanolides isolated from Xanthium macrocarpum. Fitoterapia. 2005;76:363-6.

14. Sato $Y$, Oketani $H$, Yamada T, Singyouchi K, Ohtsubo T, Kihara M, et al. A xanthanolide with potent antibacterial activity against methicillin-resistant Staphylococcus aureus. J Pharm Pharmacol. 1997;49:1042-4.

15. Tao L, Cao Y, Wei Z, Jia Q, Yu S, Zhong J, et al. Xanthatin triggers Chk1-mediated DNA damage response and destabilizes Cdc25C via lysosomal degradation in lung cancer cells. Toxicol Appl Pharmacol. 2017;337:85-94.

16. Tao L, Fan F, Liu Y, Li W, Zhang L, Ruan J, et al. Concerted suppression of STAT3 and GSK3beta is involved in growth inhibition of non-small cell lung cancer by xanthatin. PLoS One. 2013;8:e81945.

17. Takeda S, Noguchi M, Matsuo K, Yamaguchi $Y$, Kudo T, Nishimura $H$, et al. (-)-Xanthatin up-regulation of the GADD45gamma tumor suppressor gene in MDA-MB-231 breast cancer cells: role of topoisomerase llalpha inhibition and reactive oxygen species. Toxicology. 2013;305:1-9.

18. Takeda S, Nishimura H, Koyachi K, Matsumoto K, Yoshida K, Okamoto $Y$, et al. (-)-Xanthatin induces the prolonged expression of c-Fos through an $\mathrm{N}$-acetyl-Lcysteine (NAC)-sensitive mechanism in human breast cancer MDA-MB-231 cells. J Toxicol Sci. 2013;38:547-57.

19. Li WD, Wu Y, Zhang L, Yan LG, Yin FZ, Ruan JS, et al. Characterization of xanthatin: anticancer properties and mechanisms of inhibited murine melanoma in vitro and in vivo. Phytomedicine. 2013;20:865-73.

20. Zhang L, Ruan J, Yan L, Li W, Wu Y, Tao L, et al. Xanthatin induces cell cycle arrest at G2/M checkpoint and apoptosis via disrupting NF-kappaB pathway in A549 non-small cell lung cancer cells. Molecules. 2012;17:3736-50.

21. Takeda S, Matsuo K, Yaji K, Okajima-Miyazaki S, Harada M, Miyoshi $\mathrm{H}$, et al (-)-Xanthatin selectively induces GADD45gamma and stimulates caspaseindependent cell death in human breast cancer MDA-MB-231 cells. Chem Res Toxicol. 2011;24:855-65.

22. Zhang L, Tao L, Ruan J, Li W, Wu Y, Yan L, et al. Xanthatin induces G2/M cell cycle arrest and apoptosis in human gastric carcinoma MKN-45 cells. Planta Med. 2012;78:890-5.

23. Tao L, Sheng $X$, Zhang L, Li W, Wei Z, Zhu $P$, et al. Xanthatin anti-tumor cytotoxicity is mediated via glycogen synthase kinase-3beta and beta-catenin. Biochem Pharmacol. 2016;115:18-27.

24. Bi SX, Li XH, Wei CS, Xiang HH, Shen YX, Yu YQ. The antitumour growth and antiangiogenesis effects of xanthatin in murine glioma dynamically evaluated by dynamic contrast-enhanced magnetic resonance imaging. Phytother Res. 2019;33:149-58.

25. Tabas I, Ron D. Integrating the mechanisms of apoptosis induced by endoplasmic reticulum stress. Nat Cell Biol. 2011;13:184-90.

26. Bettigole SE, Glimcher LH. Endoplasmic reticulum stress in immunity. Annu Rev Immunol. 2015;33:107-38.

27. Johnson GG, White MC, Grimaldi M. Stressed to death: targeting endoplasmic reticulum stress response induced apoptosis in gliomas. Curr Pharm Des. 2011;17:284-92.

28. Obacz J, Avril T, Le Reste PJ, Urra H, Quillien V, Hetz C, et al. Endoplasmic reticulum proteostasis in glioblastoma-From molecular mechanisms to therapeutic perspectives. Sci Signal. 2017;10:pii: eaal2323.

29. Le Reste PJ, Avril T, Quillien V, Morandi X, Chevet E. Signaling the unfolded protein response in primary brain cancers. Brain Res. 2016;1642:59-69.

30. Penaranda Fajardo NM, Meijer C, Kruyt FA. The endoplasmic reticulum stress/ unfolded protein response in gliomagenesis, tumor progression and as a therapeutic target in glioblastoma. Biochem Pharmacol. 2016;118:1-8.

31. Shen $Y$, Sun A, Wang $Y$, Cha $D$, Wang $H$, Wang $F$, et al. Upregulation of mesencephalic astrocyte-derived neurotrophic factor in glial cells is associated with ischemia-induced glial activation. J Neuroinflammation. 2012;9:254. 
32. Yu YQ, Liu LC, Wang FC, Liang Y, Cha DQ, Zhang JJ, et al. Induction profile of MANF/ARMET by cerebral ischemia and its implication for neuron protection. J Cereb Blood Flow Metab. 2010;30:79-91.

33. Chen $L$, Feng $L$, Wang $X$, Du J, Chen $Y$, Yang W, et al. Mesencephalic astrocytederived neurotrophic factor is involved in inflammation by negatively regulating the NF-KB pathway. Sci Rep. 2015;5:8133.

34. Feng L, Zhang J, Zhu N, Ding Q, Zhang X, Yu J, et al. Ubiquitin ligase SYVN1/HRD1 facilitates degradation of the SERPINA1 $Z$ variant/alpha-1-antitrypsin $Z$ variant via SQSTM1/p62-dependent selective autophagy. Autophagy. 2017;13:686-702.

35. Kolb PS, Ayaub EA, Zhou W, Yum V, Dickhout JG, Ask K. The therapeutic effects of 4-phenylbutyric acid in maintaining proteostasis. Int J Biochem Cell Biol. 2015;61:45-52.

36. Mizukami T, Orihashi K, Herlambang B, Takahashi S, Hamaishi M, Okada K, et al. Sodium 4-phenylbutyrate protects against spinal cord ischemia by inhibition of endoplasmic reticulum stress. J Vasc Surg. 2010;52:1580-6.
37. Fang $X Y$, Zhang $H$, Zhao L, Tan S, Ren $Q C$, Wang $L$, et al. A new xanthatin analogue 1beta-hydroxyl-5alpha-chloro-8-epi-xanthatin induces apoptosis through ROS-mediated ERK/p38 MAPK activation and JAK2/ STAT3 inhibition in human hepatocellular carcinoma. Biochimie. 2018;152: 43-52.

38. Babaei G, Aliarab A, Abroon S, Rasmi Y, Aziz SG. Application of sesquiterpene lactone: a new promising way for cancer therapy based on anticancer activity. Biomed Pharmacother. 2018;106:239-46.

39. Wang M, Kaufman RJ. The impact of the endoplasmic reticulum protein-folding environment on cancer development. Nat Rev Cancer. 2014;14:581-97.

40. Lin $\mathrm{JH}$, Li H, Zhang $\mathrm{Y}$, Ron $\mathrm{D}$, Walter P. Divergent effects of PERK and IRE1 signaling on cell viability. PLoS One. 2009;4:e4170.

41. Puthalakath $H$, O'Reilly LA, Gunn P, Lee L, Kelly PN, Huntington ND, et al. ER stress triggers apoptosis by activating BH3-only protein Bim. Cell. 2007;129: 1337-49. 